September, 2003

\title{
Myers Effect and Tachyon Condensation
}

\author{
Yusuke Kimura \\ Theoretical Physics Laboratory, RIKEN, \\ Wako, Saitama 351-0198, Japan \\ kimuray@postman.riken.go.jp
}

\begin{abstract}
D0-branes are unstable in the presence of an R-R field strength background. A fuzzy twosphere is classically stable under such a background, this phenomenon being called the Myers effect. We analyze this effect from the viewpoint of tachyon condensation. It is explicitly shown that a fuzzy two-sphere is realized by the condensation of tachyons which appear from strings connecting different D0-branes. The formation of a fuzzy $C P^{2}$ is also investigated by considering an $S U(3)$ invariant R-R field strength background. We find that the dynamics of the D-branes depends on the properties of the associated algebra.
\end{abstract}




\section{Introduction}

The discovery of D-branes has led to exciting developments in string theory such as the relationship between Yang-Mills theory and string theory [1, 2]. The appreciation of noncommutative geometry in string theory also comes from developments of D-brane. A low energy effective action of $N$ coincident D-branes is described by the $U(N)$ Yang Mills theory, $U(N)$ adjoint scalars representing the transverse coordinates of this system [3]. Since they are given by $U(N)$ matrices, this fact suggests that the space-time probed by D-branes is related to noncommutative geometry. The idea of noncommutative geometry has found a prominent role in string theory 4 . Concrete models for investigating the nonperturbative aspects of string theory were proposed in [5, 6], and the relationship between matrix models and noncommutative geometry were argued in [7, 8, 9] for example.

Myers showed that the low energy effective action of $N$ D-branes in the presence of a constant R-R field strength background has an additional Chern-Simons term, and that the minimum of the potential is given by the configuration where transverse coordinates form a fuzzy sphere [10]. The fuzzy two-sphere has been investigated from several points of view [11, 12, 13, 14, 15, 16]. Investigating the dynamics of curved D-branes is important. The dynamic properties of a fuzzy sphere look quite different from those of a flat D-brane. This is investigated by the matrix model action with the Chern-Simons term [10]. We can summarize that D0-branes or some fuzzy spheres are unstable due to the presence of an R-R field strength background, and that only stable configuration is a fuzzy sphere which is given by the irreducible representation of $S U(2)$. A configuration composed of some fuzzy spheres and D0-branes is given by the reducible representation of $S U(2)$. This system has tachyonic instability, being considered to roll down to a fuzzy sphere which is given by the irreducible representation after tachyon condensation 17. 18. Other works investigating the dynamics of fuzzy sphere have also been reported in 19, 20, 21, 22, 23, 24, 25].

Stories of tachyon condensation have been developed on various grounds since the conjectures made by Sen [26]. Earlier works on tachyon condensation can be found in [27]. The existence of a tachyon implies instability of the perturbative vacua, condensation of the tachyon producing a stable configuration. The instability indicated by tachyons appearing around the reducible representation is also considered in this way. The irreducible representation is expected to be realized after the tachyon acquires a non-vanishing vacuum expectation value. We calculate in this paper the tachyon potentials and explicitly show the process of the tachyon condensation by using the Yang-Mills action with the Chern-Simons term. We have an extra parameter which is given by the R-R field strength in comparison to the effective action in a flat background. By tuning this parameter in such a way that the low energy Yang-Mills description of this system is valid, we can analyze the tachyon without using the string field theory. The Yang-Mills theory provides a simple field theory model that enables us to analyze the process of tachyon condensation. There have been some studies which investigated tachyon condensation by using the Yang-Mills action [28, 29, 30, 31, 32, 33, 34, 35].

The organization of this paper is as follow. Section 2 presents a matrix model with the 
Chern-Simons term and classical solutions of the model. Fuzzy spheres and D0-branes appear as classical solutions of this model, the minimum of classical energy being realized by a fuzzy sphere. In section 3, we explicitly show that the Myers effect can be viewed as the phenomenon of tachyon condensation. Tachyon potentials are obtained for some situations. In section 4 we replace the structure constant of $S U(2)$ with that of $S U(3)$ in the previous matrix model. Not only the fuzzy sphere, but also fuzzy $C P^{2}$ becomes a classical solution. We explain the concept of fuzzy $C P^{2}$, the coordinates of this space being given by the generators of the $S U(3)$ algebra. The $(m, 0)$ representation provides a four-dimensional space and the $(m, n)$ representation a six-dimensional space. D0-branes under the $S U(3)$ background are considered in section [5] An analysis analogous to that in section 3 is presented. We can observe such phenomena as the formation of the fuzzy two-sphere and the fuzzy $C P^{2}$. Moreover, interesting phenomena that the formation of the fuzzy $C P^{2}$ from D0-branes and fuzzy two-spheres can be seen due to the rich structure of the $S U(3)$ algebra. The stability of these curved branes constructed from the Lie algebra is closely related to the property of the associated algebra. Section [6] is devoted to summary and discussions. In Appendix $\mathrm{A}$ the $(m, n)$ representation of $S U(3)$ is constructed. Instability of the fuzzy sphere and fuzzy $C P^{2}$ is shown in Appendix $\mathrm{B}$ by considering the spectrum of open strings connecting a D0-brane and the curved branes.

\section{Myers effect and fuzzy sphere}

Let us first consider the effective action of $N$ D0-branes in a flat background. Such an action is given by the dimensional reduction to one dimension of $U(N)$ ten-dimensional super Yang-Mills action 3. Since we are only interested in static classical solutions in this study, the kinetic term is ignored. The potential term of this action is given by

$$
V=\lambda^{2} T_{0} \operatorname{Tr}\left(-\frac{1}{4}\left[\Phi_{\mu}, \Phi_{\nu}\right]\left[\Phi_{\mu}, \Phi_{\nu}\right]\right)
$$

where $T_{0}=1 / g_{s} l_{s}$ is the tension of a D0-brane and $\lambda=2 \pi l_{s}^{2}$. The indices take values $\mu, \nu=$ $1, \cdots, 9$. The fields $\Phi_{\mu}$ are scalar fields describing transverse fluctuations of the $N$ D0-branes. Our conventions are such that $\Phi_{\mu}$ have the dimensions of $l e n g t h^{-1}$. We now define fields which have the mass dimensions of length, $X_{\mu}=\lambda \Phi_{\mu}$. This action has two types of classical solution. One is $N$ D0-branes:

$$
\left[X_{\mu}, X_{\nu}\right]=0
$$

and the other is a flat D-brane:

$$
\left[X_{\mu}, X_{\nu}\right]=-i C_{\mu \nu} \mathbf{1}
$$

Both classical solutions are stable.

When we consider a constant R-R four form field strength background, an interesting phenomenon occurs [10]. The potential of the $N$ D0-branes has an additional Chern-Simons term ${ }^{1}$ :

$$
V=\frac{T_{0}}{\lambda^{2}} \operatorname{Tr}\left(-\frac{1}{4}\left[X_{\mu}, X_{\nu}\right]\left[X_{\mu}, X_{\nu}\right]+\frac{i}{3} \alpha \epsilon_{\mu \nu \lambda} X_{\mu}\left[X_{\nu}, X_{\lambda}\right]\right) .
$$

\footnotetext{
${ }^{1}$ We follow the notation in [10] in which the R-R charge is defined as $\mu_{0}=T_{0}$.
} 
The extrema of this potential are given by the following equations:

$$
\left[X_{\nu},\left(\left[X_{\mu}, X_{\nu}\right]-i \alpha \epsilon_{\mu \nu \lambda} X_{\lambda}\right)\right]=0 .
$$

Classical solutions which satisfy the above equations are given by $N$ D0-branes

$$
\left[X_{\mu}, X_{\nu}\right]=0
$$

or the following non-abelian solution

$$
X_{\mu}=\alpha L_{\mu}, \quad\left[L_{\mu}, L_{\nu}\right]=i \epsilon_{\mu \nu \lambda} L_{\lambda},
$$

where $L_{\mu}$ are an $N \times N$ matrix representation of the $S U(2)$ algebra. When this representation is irreducible, the solution gives a spherical D2-brane whose radius is given by the quadratic Casimir:

$$
\rho^{2}=X_{\mu} X_{\mu}=\alpha^{2} L_{\mu}^{(j)} L_{\mu}^{(j)}=\alpha^{2} j(j+1)=\alpha^{2}\left(N^{2}-1\right) / 4
$$

where $L_{\mu}^{(j)}$ denote the spin $j=(N-1) / 2$ representation of $S U(2)$. This solution has been investigated from the viewpoint of noncommutative geometry, and is called a fuzzy sphere or noncommutative sphere [12. This fuzzy sphere is composed of $N$ D0-branes and a D2-brane [10]. A smooth sphere is obtained as $N$ becomes large with $\rho$ fixed.

Reducible representations are also extrema of the potential. While the irreducible representation of $S U(2)$ represents a fuzzy sphere, the reducible representation represents some fuzzy spheres. Reducible representation of the form $L_{\mu}=\operatorname{diag}\left(L_{\mu}^{\left(j_{1}\right)}, \cdots, L_{\mu}^{\left(j_{s}\right)}\right)$ denotes $s$ fuzzy spheres. $N$ and the radii of the fuzzy spheres are given by

$$
N=\sum_{r=1}^{s} N_{r}=\sum_{r=1}^{s}\left(2 j_{r}+1\right), \quad\left(\rho_{r}^{2}\right)^{2}=\alpha^{2} j_{r}\left(j_{r}+1\right) .
$$

The classical energy of the irreducible and reducible representations is calculated as follows,

$$
\begin{aligned}
& E_{i r r}=-\frac{\alpha^{4}}{6} j(j+1)(2 j+1), \\
& E_{\text {red }}=-\frac{\alpha^{4}}{6} \sum_{r=1}^{s} j_{r}\left(j_{r}+1\right)\left(2 j_{r}+1\right) .
\end{aligned}
$$

We can find that the classical energy of the irreducible representation is less than that of the reducible representation when we fix $N$. Therefore, the reducible representations are expected to correspond to unstable extrema of the potential, collapsing into a sphere which is constructed from the irreducible representation. Instability of the reducible representations is closely related to the appearance of tachyonic modes [17, 18]. In the next section, we explicitly check that an irreducible representation is realized by the condensation of tachyons which appear from offdiagonal fluctuations.

Note that some solutions which do not belong to the $S U(2)$ algebra satisfy the equations of motion (5). An example of such solutions is a system composed of a D0-brane and a fuzzy two-sphere:

$$
X_{\mu}=\alpha\left(\begin{array}{cc}
c_{\mu} & 0 \\
0 & L_{\mu}
\end{array}\right)
$$


$c_{\mu}$ is a position of the D0-brane and $L_{\mu}$ is the spin $(N-2) / 2$ representation of $S U(2)$.

Although we consider the effective action of D0-branes, these analyses are not restricted to D0-branes. We may consider the effective action of $\mathrm{D} p$-branes such that three transverse scalars are described by the action in equation (4).

Before finishing this section, we estimate a region where these calculations are reliable. We use the Yang-Mills action in (44) to describe tachyons arising from open strings around D0-branes and spherical D2-branes. Using this action is valid when the commutator, $\lambda\left[\Phi_{\mu}, \Phi_{\nu}\right]$, is small enough [10. This condition is equivalent to $\alpha \rho=\alpha^{2} N=\rho^{2} / N \ll \lambda$. If we introduce the noncommutative scale as $l_{n c}^{2}=4 \pi \rho^{2} / N$, the condition becomes

$$
l_{n c} \ll l_{s} .
$$

It will be found that this condition ensures a suitably small tachyon mass compared to the string scale. Furthermore, we impose $g_{s} \ll 1$ to ensure the classical analysis.

\section{$3 \quad$ Fuzzy sphere from D0-branes}

In this section, we consider a system composed of some D0-branes under a constant R-R four form field strength background, explicitly showing that a fuzzy two-sphere is realized by the condensation of tachyons which arise from strings connecting different D0-branes.

We expand the matrices as

$$
X_{\mu}=\hat{x}_{\mu}+A_{\mu}
$$

where $\hat{x}_{\mu}$ is a classical solution. In this section, the solution (6) is considered. $A_{\mu}$ is the fluctuation around the classical solution, representing the fields which appear from strings connecting D0branes. Their off-diagonal components are particularly important, since they provide tachyonic modes. The potential (4) is expanded as follows:

$$
V=V_{0}+V_{2}+V_{3}+V_{4}
$$

where $V_{0}$ is the classical energy, and

$$
\begin{aligned}
V_{2} & =\frac{1}{2} \operatorname{Tr}\left(A_{\nu}\left[\hat{x}_{\mu},\left[\hat{x}_{\mu}, A_{\nu}\right]\right]\right)+2 \operatorname{Tr}\left(A_{\mu}\left(\left[\hat{x}_{\mu}, \hat{x}_{\nu}\right]-i \alpha \epsilon_{\mu \nu \lambda} \hat{x}_{\lambda}\right) A_{\nu}\right), \\
V_{3} & =-\operatorname{Tr}\left(\left[\hat{x}_{\mu}, A_{\nu}\right]\left[A_{\mu}, A_{\nu}\right]\right)+\frac{i}{3} \alpha \epsilon_{\mu \nu \lambda} \operatorname{Tr}\left(A_{\mu}\left[A_{\nu}, A_{\lambda}\right]\right) \\
V_{4} & =-\frac{1}{4} \operatorname{Tr}\left(\left[A_{\mu}, A_{\nu}\right]\left[A_{\mu}, A_{\nu}\right]\right) .
\end{aligned}
$$

$V_{2}$ gives mass terms, and $V_{3}$ and $V_{4}$ give interaction terms. Note that we added the following term to fix the $U(N)$ gauge invariance,

$$
-\frac{1}{2} \operatorname{Tr}\left(\left[\hat{x}_{\mu}, A_{\mu}\right]\left[\hat{x}^{\nu}, A^{\nu}\right]\right)
$$

The overall factor in front of the potential (44) has been ignored since it is not important in the present discussion. We first show that tachyons appear from the off-diagonal components of the 
fluctuations. We then calculate $V_{3}$ and $V_{4}$ for the tachyonic fields and search for a stable extremum in which the tachyonic fields have non-zero expectation values. Finally, $X_{\mu}$ can be expected to satisfy the algebra of a fuzzy two-sphere. Similar calculations for brane and anti-brane system have been presented in [33, 34].

We first study a system of two D0-branes as the simplest case. We choose the positions of the D0-branes as follows:

$$
\hat{x}_{1}=\hat{x}_{2}=0, \quad \hat{x}_{3}=\left(\begin{array}{cc}
x_{3}^{(1)} & 0 \\
0 & x_{3}^{(2)}
\end{array}\right) \quad\left(x_{3}^{(1)} \geq x_{3}^{(2)}\right) .
$$

This choice is without loss of generality. Since the potential (44) has the translation invariance

$$
\delta X_{\mu}=c_{\mu} \mathbf{1}_{N}
$$

we restrict $X_{\mu}$ to be traceless by assuming the condition $x_{3}^{(1)}+x_{3}^{(2)}=0$. Fields appearing in this system are introduced as

$$
A_{\mu}=\left(\begin{array}{cc}
0 & a_{\mu} \\
\bar{a}_{\mu} & 0
\end{array}\right)
$$

where $\bar{a}$ is a complex conjugate of $a$. The off-diagonal components represent fields which appear from strings connecting different D-branes, and are considered to play an important role in noncommutative configurations. We now investigate a situation where noncommutative configurations are constructed after tachyons appearing from the off-diagonal parts condense. We do not turn on the diagonal components since they do not give tachyonic modes. These components are actually set to zero by using the equations of motion for them. Although there may be possibility that the diagonal comonents can have nonzero values due to interactions with off-diagonal fields, we first assume that the diagonal parts do not contribute to noncommutative solutions to simplify the calculation. A noncommutative (fuzzy sphere) solution is actually obtained by considering only off-diagonal parts.

We can easily calculate the mass term of the off-diagonal components as follows,

$$
\begin{aligned}
V_{2} & =a_{\mu}\left(\delta_{\mu \nu} \Delta^{2}+2 i \alpha \epsilon_{\mu \nu 3} \Delta\right) \bar{a}_{\nu} \\
& =\left(\Delta^{2}-2 \alpha \Delta\right) a_{+} \bar{a}_{-}+\left(\Delta^{2}+2 \alpha \Delta\right) a_{-} \bar{a}_{+}+\Delta^{2} a_{3} \bar{a}_{3}
\end{aligned}
$$

where $\Delta \equiv x_{3}^{(1)}-x_{3}^{(2)} \geq 0$ and

$$
a_{+} \equiv \frac{1}{\sqrt{2}}\left(a_{1}+i a_{2}\right), \quad \bar{a}_{ \pm} \equiv \frac{1}{\sqrt{2}}\left(\bar{a}_{1} \pm i \bar{a}_{2}\right)
$$

Note that $\bar{a}_{\mp}$ are complex conjugate of $a_{ \pm}$. These fields are complex, since the strings connecting different D-branes are orientable. Since $\Delta$ is positive, only $a_{+}$can be tachyonic. A complex tachyon appears when the distance between two D-branes is short, $0<\Delta<2 \alpha$. We are interested in this case. Non-tachyonic modes are set to zero $a_{-}=a_{3}=0$ (by using the equations of motion), and we parameterize $a_{+}$as

$$
a_{+}=t e^{i \theta}
$$


where $t \geq 0$ and $0 \leq \theta<2 \pi$. The full potential ${ }^{2}$ is calculated as

$$
V=\left(\Delta^{2}-2 \alpha \Delta\right) t^{2}+t^{4} \equiv m^{2} t^{2}+t^{4}
$$

This potential does not depend on $\theta$. This fact reflects the $U(1)$ symmetry of this system which is related to the rotation around the 3 -axis. We look for extrema of this potential which satisfy $d V / d t=0$. It should be noted that condition $d V / d t=0$ is equivalent to the condition that $X_{\mu}$ satisfy the equations of motion (5D). The point $t=0$ corresponding to two D0-branes is an unstable extremum. The value of $t$ corresponding to a stable extremum is found to be

$$
t^{2}=-\frac{1}{2} m^{2}
$$

At this point, $V$ takes the following value,

$$
V=-\frac{1}{4} m^{4}=-\frac{1}{4}\left(\Delta^{2}-2 \alpha \Delta\right)^{2},
$$

which is lower than the classical potential energy of two D0-branes. We next look for a configuration which minimizes this potential. It is minimized when $\Delta=\alpha$. In this case, the tachyon mass and the classical energy of this configuration are $m^{2}=-\alpha^{2}$ and $V=-\alpha^{4} / 4$. This value of $V$ is indeed the classical energy of a fuzzy sphere which is constructed from the $j=1 / 2$ representation of $S U(2)$ (see (10)). We can easily recognize that matrices $X_{\mu}$ can be written as $X_{\mu}=\alpha \sigma_{\mu} / 2^{3}$.

The tachyon mass is given by

$$
m_{t}^{2}=-\frac{\alpha^{2}}{l_{s}^{4}}=-\frac{l_{n c}^{2}}{N l_{s}^{2}} \frac{1}{l_{s}^{2}}
$$

after restoring the string scale. The restriction (13) ensures that this tachyon mass is much smaller than the string mass scale.

Let us now investigate what configuration is realized when the tachyon condenses to another value. If we fix the distance between two D0-branes as $\Delta=\alpha / 2$ for example, the tachyon potential becomes $V=-\frac{3}{4} \alpha^{2} t^{2}+t^{4}$. Then the tachyon can condense at $t^{2}=3 \alpha^{2} / 8$, realizing the following solution:

$$
X_{1}=\frac{\sqrt{3}}{4} \alpha \sigma_{1}, \quad X_{2}=\frac{\sqrt{3}}{4} \alpha \sigma_{2}, \quad X_{3}=\frac{\alpha}{4} \sigma_{3} .
$$

A fuzzy ellipsoidal sphere is realized. The energy of this solution is $V=-9 \alpha^{4} / 64$, which is higher than that of a fuzzy two-sphere. By comparing this solution with a fuzzy two-sphere solution, we find that a solution which has higher symmetry has lower energy.

We next argue a system of three D0-branes whose positions are

$$
\hat{x}_{1}=\hat{x}_{2}=0, \quad \hat{x}_{3}=\left(\begin{array}{ccc}
x_{3}^{(1)} & 0 & 0 \\
0 & x_{3}^{(2)} & 0 \\
0 & 0 & x_{3}^{(3)}
\end{array}\right) .
$$

\footnotetext{
${ }^{2}$ The interaction term $V_{3}$ is zero in the present situations, while it is not zero in section 5

${ }^{3}$ We have set $\theta=0$ in this expression.
} 
$x_{3}^{(i)}$ are restricted to $x_{3}^{(1)} \geq x_{3}^{(2)} \geq x_{3}^{(3)}$ and $x_{3}^{(1)}+x_{3}^{(2)}+x_{3}^{(3)}=0$ without loss of generality. We denote fluctuations by

$$
A_{\mu}=\left(\begin{array}{ccc}
0 & a_{\mu} & b_{\mu} \\
\bar{a}_{\mu} & 0 & c_{\mu} \\
\bar{b}_{\mu} & \bar{c}_{\mu} & 0
\end{array}\right) .
$$

The calculation is analogous to the previous case. The mass terms of these fields are

$$
\begin{aligned}
V_{2}= & a_{\mu}\left(\delta_{\mu \nu} \Delta_{12}^{2}+2 i \alpha \epsilon_{\mu \nu 3} \Delta_{12}\right) \bar{a}_{\nu} \\
& +b_{\mu}\left(\delta_{\mu \nu} \Delta_{13}^{2}+2 i \alpha \epsilon_{\mu \nu 3} \Delta_{13}\right) \bar{b}_{\nu}+c_{\mu}\left(\delta_{\mu \nu} \Delta_{23}^{2}+2 i \alpha \epsilon_{\mu \nu 3} \Delta_{23}\right) \bar{c}_{\nu} \\
= & \left(\Delta_{12}^{2}-2 \alpha \Delta_{12}\right) a_{+} \bar{a}_{-}+\left(\Delta_{12}^{2}+2 \alpha \Delta_{12}\right) a_{-} \bar{a}_{+}+\Delta_{12}^{2} a_{3} \bar{a}_{3} \\
& +\left(\Delta_{13}^{2}-2 \alpha \Delta_{13}\right) b_{+} \bar{b}_{-}+\left(\Delta_{13}^{2}+2 \alpha \Delta_{13}\right) b_{-} \bar{b}_{+}+\Delta_{13}^{2} b_{3} \bar{b}_{3} \\
& +\left(\Delta_{23}^{2}-2 \alpha \Delta_{23}\right) c_{+} \bar{c}_{-}+\left(\Delta_{23}^{2}+2 \alpha \Delta_{23}\right) c_{-} \bar{c}_{+}+\Delta_{23}^{2} c_{3} \bar{c}_{3},
\end{aligned}
$$

where $\Delta_{i j}=x_{3}^{(i)}-x_{3}^{(j)} \cdot a_{ \pm}$are defined in (22),$b_{ \pm}$and $c_{ \pm}$being analogously defined. Since $\Delta$ is positive, three complex tachyon fields can appear when $0<\Delta<2 \alpha$. After dropping the fields except $a_{+}, b_{+}$and $c_{+}, V_{4}$ can be evaluated as follows,

$$
\begin{aligned}
V_{4}= & \left(a_{+} \bar{a}_{-}\right)^{2}+\left(b_{+} \bar{b}_{-}\right)^{2}+\left(c_{+} \bar{c}_{-}\right)^{2} \\
& -\left(a_{+} \bar{a}_{-}\right)\left(c_{+} \bar{c}_{-}\right)+2\left(a_{+} \bar{a}_{-}\right)\left(b_{+} \bar{b}_{-}\right)+2\left(b_{+} \bar{b}_{-}\right)\left(c_{+} \bar{c}_{-}\right) .
\end{aligned}
$$

Then the full potential for those fields takes the following form

$$
V=m_{1}^{2} t_{1}^{2}+m_{2}^{2} t_{2}^{2}+m_{3}^{2} t_{3}^{2}+t_{1}^{4}+t_{2}^{4}+t_{3}^{4}-t_{1}^{2} t_{2}^{2}+2 t_{1}^{2} t_{3}^{2}+2 t_{2}^{2} t_{3}^{2},
$$

where $t_{i}$ and $m_{i}$ are defined as

$$
a_{+}=t_{1} e^{i \theta_{1}}, \quad c_{+}=t_{2} e^{i \theta_{2}}, \quad b_{+}=t_{3} e^{i \theta_{3}},
$$

and

$$
m_{1}=\Delta_{12}^{2}-2 \alpha \Delta_{12}, \quad m_{2}=\Delta_{23}^{2}-2 \alpha \Delta_{23}, \quad m_{3}=\Delta_{13}^{2}-2 \alpha \Delta_{13} .
$$

This is also independent of $\theta$, reflecting the $U(1)$ symmetry. The point corresponding to the condensation of tachyons is given by a stable extremum of the potential. This can be found by solving the following equations,

$$
\begin{aligned}
& \frac{d V}{d t_{1}}=\left(m_{1}^{2}+2 t_{1}^{2}-t_{2}^{2}+2 t_{3}^{2}\right) t_{1}=0 \\
& \frac{d V}{d t_{2}}=\left(m_{2}^{2}+2 t_{2}^{2}-t_{1}^{2}+2 t_{3}^{2}\right) t_{2}=0 \\
& \frac{d V}{d t_{3}}=\left(m_{3}^{2}+2 t_{3}^{2}+2 t_{1}^{2}+2 t_{2}^{2}\right) t_{3}=0 .
\end{aligned}
$$

The point $t_{1}=t_{2}=t_{3}=0$ is a trivial solution, which represents an unstable configuration. We have to search for a point corresponding to a local minimum. Since solutions of these equations depend on the values of $\Delta_{12}, \Delta_{23}$ and $\Delta_{13}$, the problem is complicated. We therefore consider the following situation to simplify the analysis,

$$
\Delta_{23}=\alpha, \quad \Delta_{12} \equiv \Delta>0, \quad \Delta_{13}=\Delta+\alpha .
$$


The mass (35) can then be rewritten in terms of $\Delta$ as

$$
m_{1}^{2}=\Delta(\Delta-2 \alpha), \quad m_{2}^{2}=-\alpha^{2}, m_{3}^{2}=(\Delta+\alpha)(\Delta-\alpha) .
$$

$m_{2}^{2}$ is always negative, and $m_{1}^{2}$ and $m_{3}^{2}$ can be negative when $0<\Delta<2 \alpha$ and $0<\Delta<\alpha$ respectively. We have to consider the following three cases:

$$
\text { I: } \quad 0<\Delta<\alpha, \quad \text { II: } \quad \alpha \leq \Delta<\Delta_{0}, \quad \text { III: } \quad \Delta_{0} \leq \Delta \text {. }
$$

It will be clear soon why we have introduced $\Delta_{0}$. Let us first consider the situation where $\Delta$ is large enough (case III). The definition of $\Delta_{0}$ is such that only $t_{2}$ is tachyonic in the case III. The tachyon potential is given by

$$
V=-\alpha^{2} t_{2}^{2}+t_{2}^{4}
$$

Note that this potential does not depend on $\Delta$. If we change the position of the first D0-brane such that $\Delta \geq \Delta_{0}$, the form of this potential does not change. At the minimum of this potential $t_{2}^{2}=\alpha^{2} / 2$, the following configuration is realized:

$$
X_{1}=\left(\begin{array}{cc}
0 & \\
& \frac{\alpha}{2} \sigma_{1}
\end{array}\right), X_{2}=\left(\begin{array}{cc}
0 & \\
& \frac{\alpha}{2} \sigma_{2}
\end{array}\right), X_{3}=\left(\begin{array}{cc}
c & \\
& \frac{\alpha}{2} \sigma_{3}-d 1_{2}
\end{array}\right) .
$$

where $c=\frac{\alpha}{3}+\frac{2}{3} \Delta$ and $d=\frac{\alpha}{6}+\frac{1}{3} \Delta$. A D0-brane is in the position $x_{3}=c$, while a spherical D2brane which is given by the spin-1/2 representation of $S U(2)$ is in the position $x_{3}=-d$. Though this matrix $X_{\mu}$ does not satisfy the $\mathrm{SU}(2)$ algebra, it is a solution of the equations of motion (5).

We next consider case II, and determine the value of $\Delta_{0}$. Since $m_{2}^{2}$ is always negative, $t_{2}$ can always take a non-zero solution in (36). On the other hand, whether $t_{1}$ can take a non-zero value or not depends on $\Delta$. Naively, $t_{1}$ is zero in a case $\Delta \geq 2 \alpha$ because $m_{1}^{2}$ is positive. We must draw attention to the existence of the interaction term $-t_{1}^{2} t_{2}^{2}$ in (33). $\Delta_{0}$ is not $2 \alpha$ due to this term $\left(\Delta_{0}\right.$ is larger than $\left.2 \alpha\right)$. We now require that both $t_{1}$ and $t_{2}$ can condense to non-zero values. The potential for them is

$$
V=m_{1}^{2} t_{1}^{2}+t_{1}^{4}-\alpha^{2} t_{2}^{2}+t_{2}^{4}-t_{1}^{2} t_{2}^{2} .
$$

From the equations of motion (36), tachyons $t_{1}$ and $t_{2}$ respectively can condense at the following values,

$$
\begin{aligned}
t_{1}^{2} & =-\frac{2}{3} m_{1}^{2}-\frac{1}{3} m_{2}^{2}=-\frac{2}{3}(\Delta-\alpha)^{2}+\alpha^{2} \\
t_{2}^{2} & =-\frac{1}{3} m_{1}^{2}-\frac{2}{3} m_{2}^{2}=-\frac{1}{3}(\Delta-\alpha)^{2}+\alpha^{2} .
\end{aligned}
$$

$\Delta_{0}$ is determined by the condition that both $t_{1}^{2}$ and $t_{2}^{2}$ have to be positive. The conditions $t_{1}^{2}>0$ and $t_{2}^{2}>0$ provide $0<\Delta<\left(1+\sqrt{\frac{3}{2}}\right) \alpha$ and $0<\Delta<(1+\sqrt{3}) \alpha$ respectively. Therefore $\Delta_{0}$ needs to be $\left(1+\sqrt{\frac{3}{2}}\right) \alpha$. We then substitute $t_{1}^{2}$ and $t_{2}^{2}$ into $V$. It can be found, after some calculations, that $V$ is minimized when $\Delta=\alpha$, that is

$$
t_{1}=\alpha, \quad t_{2}=\alpha, \quad t_{3}=0
$$


The value of $V$ is

$$
V=-\alpha^{4}
$$

It can be easily checked that $X_{\mu}$ satisfy the spin-1 representation of $S U(2)$. A fuzzy sphere is obtained.

In case I, three fields are tachyonic, which can condense to the following values,

$$
t_{1}^{2}=-\frac{2}{3} m_{1}^{2}-\frac{1}{3} m_{2}^{2}, \quad t_{2}^{2}=-\frac{1}{3} m_{1}^{2}-\frac{2}{3} m_{2}^{2}, \quad t_{3}=0 .
$$

where $m_{1}^{2}$ and $m_{2}^{2}$ are given by (38). This point seems to be unstable, since $t_{3}$ is zero. It is, however, stable since the interaction terms $\left\langle t_{1}^{2}\right\rangle t_{3}^{2}$ and $\left\langle t_{2}^{2}\right\rangle t_{3}^{2}$ in the potential (33) can give the mass of $t_{3}$. The stability of this point can be easily confirmed as $d^{2} V / 2 d t_{1}^{2}=-\frac{8}{3} m_{1}^{2}-\frac{4}{3} m_{2}^{2}>0$, $d^{2} V / 2 d t_{2}^{2}=-\frac{4}{3} m_{1}^{2}-\frac{8}{3} m_{2}^{2}>0$ and $d^{2} V / 2 d t_{3}^{2}=m_{3}^{2}-2 m_{1}^{2}-2 m_{2}^{2}>0$. The configuration that is realized in this case is one which is similar to a fuzzy ellipsoidal sphere (28).

It is worth while examining the D0-D2 system (40) more closely. This system is stable whenever the position of the D0-brane is $\Delta \geq \Delta_{0}$. As has already been investigated [17, 18, this system becomes unstable when the D0-brane is close to the surface of the spherical D2-brane. From equation (B.4), a tachyonic mode appears when $\Delta$ takes the following interval,

$$
\frac{3}{2}-\sqrt{\frac{3}{2}}<\frac{1}{2}+\frac{\Delta}{\alpha}<\frac{3}{2}+\sqrt{\frac{3}{2}} .
$$

The upper bound of $\Delta$ is equivalent to $\Delta_{0}$. This system is stable when the D0-brane is far enough from the D2-brane. As we decrease the distance between the D0-brane and the D2-brane, the system becomes unstable and a fuzzy sphere of larger radius is formed. We will explicitly show this phenomenon from the viewpoint of tachyon condensation.

The D0-D2 system is unstable when $\Delta$ satisfies (46), and the tachyon arising from this system can be analyzed by considering the tachyon potential shown in (41). The point specified by $t_{1}=0$ and $t_{2}=\alpha / \sqrt{2}$ is an unstable extremum, the configuration expressed in (40) being realized. Let us expand the potential around this point,

$$
t_{1}=0+v, \quad t_{2}=\frac{\alpha}{\sqrt{2}}+a .
$$

Fields $v$ and $a$ represent fluctuations around the expression in (40):

$$
X_{\mu}=\left(\begin{array}{cc}
c_{\mu} & \\
& \frac{\alpha}{2} \sigma_{\mu}-d_{\mu} 1_{2}
\end{array}\right)+\left(\begin{array}{cc}
0 & v_{\mu} \\
v_{\mu}^{\dagger} & a_{\mu}
\end{array}\right) .
$$

where $v_{1}=\left(\frac{1}{\sqrt{2}} v, 0\right), v_{2}=\left(\frac{-i}{\sqrt{2}} v, 0\right), a_{1}=\frac{1}{\sqrt{2}} a \sigma_{1}$ and $a_{2}=\frac{1}{\sqrt{2}} a \sigma_{2} . \quad a_{\mu}$ is a gauge field of the noncommutative gauge theory on the fuzzy sphere [16]. The potential (41) is expressed in terms of $v$ and $a$ as follows,

$$
V=-\frac{\alpha^{4}}{4}+2 \alpha^{2} a^{2}+2 \sqrt{2} \alpha a^{3}+a^{4}+\left(m_{1}^{2}-\frac{\alpha^{2}}{2}\right) v^{2}+v^{4}-\sqrt{2} \alpha a v^{2}-v^{2} a^{2} .
$$


The mass term for $v$ is $\left(\Delta^{2}-2 \alpha \Delta-\alpha^{2} / 2\right)$ and becomes negative when $\Delta$ satisfies (46). Although the mass of $a$ is positive, it can be negative as will be shown later because of the interaction term $-v^{2} a^{2}$. The condition $d V / d v=0$ determines the extrema:

$$
\left(4 v^{2}-2 a^{2}-2 \sqrt{2} \alpha a-3 \alpha^{2}\right) v=0 .
$$

Since we want an extremum where $v$ takes a non-zero value, we substitute $4 v^{2}=2 a^{2}+2 \sqrt{2} \alpha a+3 \alpha^{2}$ into (49). Finally we get the following potential for $a$ :

$$
V=-\frac{13}{16} \alpha^{4}-\frac{3 \sqrt{2}}{4} \alpha^{3} a+\frac{3}{4} \alpha^{2} a^{2}+\frac{3 \sqrt{2}}{2} \alpha a^{3}+\frac{3}{4} a^{4} .
$$

The extrema of this potential are obtained from $d V / d a=0$ as

$$
3\left(\frac{a}{\alpha}+1+\frac{1}{\sqrt{2}}\right)\left(\frac{a}{\alpha}+\frac{1}{\sqrt{2}}\right)\left(\frac{a}{\alpha}-1+\frac{1}{\sqrt{2}}\right)=0 .
$$

The solution $a=(1-1 / \sqrt{2}) \alpha$ is what we are looking for. $v$ is determined as $v=1$. Substituting these values into equation (48), $X_{\mu}$ provide the coordinates of the fuzzy sphere which is given by the spin-1 representation of $S U(2)$. We have thus shown that a system comprising a D0-brane and a fuzzy sphere which is given by the spin- $1 / 2$ representation collapses into a fuzzy sphere which is given by the spin-1 representation after the condensation of tachyon appearing from string connecting the D0- and D2-brane.

A system composed of $N$ D0-branes can be also analogously analyzed. To simplify the calculation, we start from the following configuration:

$$
\hat{x}_{1}=\hat{x}_{2}=0, \quad \hat{x}_{3}=\left(\begin{array}{cccc}
x_{3}^{(1)} & & & \\
& x_{3}^{(2)} & & \\
& & \ddots & \\
& & & x_{3}^{(N)}
\end{array}\right)
$$

where $\Delta_{i j}=x_{3}^{(i)}-x_{3}^{(j)} \geq 0$. It is sufficient to consider the following fluctuations,

$$
A_{+}=\left(\begin{array}{cccc}
0 & a_{+}^{(1)} & & \\
& 0 & a_{+}^{(2)} & \\
& & \ddots & a_{+}^{(N-1)} \\
& & & 0
\end{array}\right), \quad A_{-}=\left(\begin{array}{cccc}
0 & & & \\
\bar{a}_{-}^{(1)} & 0 & & \\
& \bar{a}_{-}^{(2)} & \ddots & \\
& & \bar{a}_{-}^{(N-1)} & 0
\end{array}\right) \text {. }
$$

The mass terms are

$$
V_{2}=m_{1}^{2}\left(a_{+}^{(1)} \bar{a}_{-}^{(1)}\right)+m_{2}^{2}\left(a_{+}^{(2)} \bar{a}_{-}^{(2)}\right)+\cdots+m_{1}^{N-1}\left(a_{+}^{(N-1)} \bar{a}_{-}^{(N-1)}\right),
$$

where $m_{i}^{2}=\Delta_{i, i+1}\left(\Delta_{i, i+1}-2 \alpha\right)$, and interaction terms are

$$
V_{4}=2\left(a_{+}^{(1)} \bar{a}_{-}^{(1)}\right)^{2}+\cdots+2\left(a_{+}^{(N-1)} \bar{a}_{-}^{(N-1)}\right)^{2}
$$




$$
-2\left(a_{+}^{(1)} \bar{a}_{-}^{(1)}\right)\left(a_{+}^{(2)} \bar{a}_{-}^{(2)}\right)-2\left(a_{+}^{(2)} \bar{a}_{-}^{(2)}\right)\left(a_{+}^{(3)} \bar{a}_{-}^{(3)}\right) \cdots-2\left(a_{+}^{(N-2)} \bar{a}_{-}^{(N-2)}\right)\left(a_{+}^{(N-1)} \bar{a}_{-}^{(N-1)}\right) .
$$

We then have

$$
V=m_{1}^{2} t_{1}^{2}+\cdots+m_{N-1}^{2} t_{N-1}^{2}+t_{1}^{4}+\cdots+t_{n-1}^{4}-\left(t_{1}^{2} t_{2}^{2}+\cdots+t_{N-2}^{2} t_{N-1}^{2}\right) .
$$

Minimizing the tachyon potential gives $\Delta_{i, i+1}=\alpha$ for all $i$, and

$$
t_{1}=\frac{\alpha}{2} f(j-1), \quad t_{2}=\frac{\alpha}{2} f(j-2), \ldots t_{N-1}=\frac{\alpha}{2} f(-j),
$$

where $f(m)=\sqrt{j(j+1)-m(m+1)})$. This solution surely provides the spin $(N-1) / 2$ representation of $S U(2)$.

\section{$4 \quad$ Fuzzy $C P^{2}$}

We investigated in the previous section the process for the formation of a fuzzy sphere under the $S U(2)$ invariant R-R four form background from the viewpoint of tachyon condensation. The reducible representations of $S U(2)$ (and D0-branes) are basically unstable, forming the irreducible representation of $\mathrm{SU}(2)$ by the condensation of tachyons which arise from strings between the different D-branes. In this section, we consider what phenomena happen when we replace the structure constant of $S U(2)$ with that of $S U(3)$. Such matrix models have previously been investigated in [37, 42. Since the structure of $S U(3)$ is richer than that of $S U(2)$, in addition to the fact that the $S U(3)$ algebra contains the $S U(2)$ algebra as subalgebra, the dynamics of D-branes under this background becomes more complicated than that in the previous section.

The potential we will consider is as follows:

$$
V=\frac{T_{0}}{\lambda^{2}} \operatorname{Tr}\left(-\frac{1}{4}\left[X_{\mu}, X_{\nu}\right]\left[X_{\mu}, X_{\nu}\right]+\frac{i}{3} \alpha f_{\mu \nu \lambda} X_{\mu}\left[X_{\nu}, X_{\lambda}\right]\right) \quad(\mu, \nu=1, \ldots, 8) .
$$

Since we need eight transverse coordinates, this can be realized only in $\mathrm{D} p(p \leq 1)$-branes. Static classical solutions of this action are given by the following equations,

$$
\left[X_{\nu},\left(\left[X_{\mu}, X_{\nu}\right]-i \alpha f_{\mu \nu \lambda} X_{\lambda}\right)\right]=0
$$

The main classical solutions involve $N$ D0-branes

$$
\left[X_{\mu}, X_{\nu}\right]=0
$$

fuzzy two-sphere

$$
\left[X_{\mu}, X_{\nu}\right]=i \alpha \epsilon_{\mu \nu \lambda} X_{\lambda} \quad(\mu, \nu=1, \ldots, 3)
$$

and fuzzy $C P^{2}$

$$
\left[X_{\mu}, X_{\nu}\right]=i \alpha f_{\mu \nu \lambda} X_{\lambda} \quad(\mu, \nu=1, \ldots, 8) .
$$

Before studying the dynamics of these branes, we will study the last solution. 
The coordinates of fuzzy $C P^{2}$ are provided by

$$
\hat{x}_{\mu}=\alpha T_{\mu}^{(m, n)}
$$

where $T_{\mu}^{(m, n)}$ are the generators of the $(m, n)$ representation of $S U(3)$. They are realized by matrices whose size is

$$
N=\frac{1}{2}(m+1)(n+1)(m+n+2)
$$

Therefore the value of $N$ is restricted to realize this noncommutative solution, which is not the case in the fuzzy two-sphere. There have been some reports [36, 37, 38, 39, 40, 41, 42, 43, investigating such a noncommutative space. The radius of this space is given by using the formula (A.6),

$$
\rho^{2}=\hat{x}_{\mu} \hat{x}_{\mu}=\alpha^{2} T_{\mu} T_{\mu}=\alpha^{2} C_{2}(m, n)
$$

This space has $S U(3)$ isometry. There are two manifolds whose isometry is $S U(3)$, that is, $S U(3) / U(2)$ and $S U(3) / U(1) \times U(1)$. The noncommutative space which is related to the $(m, 0)$ or $(0, n)$ representation of $S U(3)$ is $S U(3) / U(2)=C P^{2}$, while the noncommutative space which is related to the $(m, m)$ representation of $S U(3)$ is $S U(3) / U(1) \times U(1)$. The latter space is locally $C P^{2} \times S^{2} . T_{8}$ is usually diagonalized as follows,

$$
\begin{aligned}
& T_{8}^{(m, 0)}=\frac{1}{2 \sqrt{3}} \operatorname{diag}\left(m \mathbf{1}_{\mathbf{m}+\mathbf{1}},(m-3) \mathbf{1},(m-6) \mathbf{1}, \cdots,-2 m\right), \\
& T_{8}^{(m, n)}=\frac{1}{2 \sqrt{3}} \operatorname{diag}\left((m+2 n) \mathbf{1}_{\mathbf{m}+\mathbf{1}}, \cdots,(-2 m-n) \mathbf{1}_{\mathbf{n}+\mathbf{1}}\right) .
\end{aligned}
$$

The eigenvalues of $T_{8}^{(m, n)}$ are arranged in order of magnitude. We introduce the south pole by the point $T_{8}^{(m, 0)}=-m / \sqrt{3}$ and $T_{8}^{(m, n)}=-(m+n / 2) / \sqrt{3}(m>n)$ in each representation. We can evaluate the quadratic Casimir of the $S U(2)$ algebra at the south pole, which is a subalgebra of the $S U(3)$ algebra ${ }^{4}$ as

$$
\begin{aligned}
& \sum_{\mu=1}^{3} T_{\mu}^{(m, 0)} T_{\mu}^{(m, 0)}=0, \\
& \sum_{\mu=1}^{3} T_{\mu}^{(m, n)} T_{\mu}^{(m, n)}=\frac{n(n+2)}{4} .
\end{aligned}
$$

This shows that the $(m, n)$ representation has a fuzzy two-sphere, which is given by the spin $n / 2$ representation, at this point. The manifold which is constructed from the $(m, n)$ representation of $S U(3)$ is locally $C P^{2} \times S^{2}$, although it is not globally the case. We can summarize as follows. The $S U(3)$ algebra gives noncommutative manifolds whose isometry is $S U(3)$. There are two choices of stability group, $U(2)$ or $U(1) \times U(1)$. It must be noted that each manifold is a symplectic manifold (see 44] for example).

The noncommutative coordinates can be realized by the guiding center coordinates on an ordinary commutative space in a magnetic monopole at the origin. The quantum Hall system on

\footnotetext{
${ }^{4}$ We used the formulae in Appendix $\mathrm{A}$ in this calculation.
} 
$C P^{2}$ was constructed in [40. Such a system is constructed in two ways; one is given by $U(1)$ gauge field background, and the other by combined $U(1)$ and $S U(2)$. These systems respectively correspond to the fuzzy spaces which are constructed by the $(m, 0)$ or $(m, n)$ representation of $S U(3)$. Although the latter fuzzy space has an extra two-dimensional space, we may call it fuzzy $C P^{2}$ since it is realized by considering a commutative $C P^{2}$ and a magnetic monopole field.

As has been shown in the previous section, the approach of a D0-brane to the surface of a fuzzy two-sphere induces instability. Analogous instability happens when a D0 brane approaches the surface of a fuzzy $C P^{2}$. The mass spectrum of the $0-4$ string is calculated in Appendix $\mathbb{B}$, and it is shown that tachyonic modes appear from the $0-4$ string. The fuzzy $C P^{2}$ basically has analogous instability to that of the fuzzy $S^{2}$.

Before going to the next section, we will comment on the classical energy of these fuzzy spaces. It is found that the energy of the $(m, 0)$ representation of $S U(3)$ is lower than that of the $\left(m^{\prime}, n^{\prime}\right)$ representation for fixed $N$ 42]. We next compare the classical energy of the fuzzy two-sphere with that of the fuzzy $C P^{2}$. Since the energy of the irreducible representation is lower than that of the reducible representation, we consider only the irreducible representation. The energy is given by

$$
\begin{gathered}
E_{S^{2}}=-\frac{\alpha^{4}}{6} j(j+1)(2 j+1), \\
E_{C P^{2}}=-\frac{\alpha^{4}}{4} C_{2}(m, n) N(m, n),
\end{gathered}
$$

where $j=(N-1) / 2$. We can find that $E_{S^{2}}$ is lower than $E_{C P^{2}}$ for fixed $N$. Therefore, if we consider some D0-branes under the $S U(3)$ invariant R-R four form background, a classical vacuum is realized by a fuzzy two-sphere configuration.

\section{$5 \quad$ Fuzzy sphere and fuzzy $C P^{2}$ from D0-branes}

In this section, we consider some D0-branes under the $S U(3)$ invariant $\mathrm{R}-\mathrm{R}$ four form background. It can be expected that the fuzzy sphere and the fuzzy $C P^{2}$ are formed by the condensation of tachyons. (The last argument presented in the previous section showed that a configuration minimizing the potential is realized by the irreducible representation of $S U(2)$. ) The formation of these branes depends on the positions of the D0-branes. We will approach this analysis by considering several situations.

We will first consider two D0-branes. The positions of them are

$$
\hat{x}_{i}=0(i \neq 3), \quad \hat{x}_{3}=\left(\begin{array}{cc}
x_{3}^{(1)} & 0 \\
0 & x_{3}^{(2)}
\end{array}\right) \quad\left(x_{3}^{(1)}-x_{3}^{(2)} \equiv \Delta>0\right),
$$

and fields which appear from strings connecting these branes are presented by

$$
A_{\mu}=\left(\begin{array}{cc}
0 & a_{\mu} \\
\bar{a}_{\mu} & 0
\end{array}\right)
$$


For later convenience, we can calculate the mass terms in more general cases of $\Delta_{3} \neq 0$ and $\Delta_{8} \neq 0$ :

$$
\begin{aligned}
V_{2}= & \frac{1}{2} \operatorname{Tr}\left(A_{\nu}\left[\hat{x}_{\mu},\left[\hat{x}_{\mu}, A_{\nu}\right]\right]\right)+2 \operatorname{Tr}\left(A_{\mu}\left(\left[\hat{x}_{\mu}, \hat{x}_{\nu}\right]-i \alpha f_{\mu \nu \lambda} \hat{x}_{\lambda}\right) A_{\nu}\right) \\
= & a_{\mu}\left(\delta_{\mu \nu} \Delta^{2}+2 i \alpha f_{\mu \nu \lambda} \Delta^{\lambda}\right) \bar{a}_{\nu} \\
= & \left(\Delta^{2}-2 \alpha \Delta_{3}\right) a_{+}^{(1)} \bar{a}_{-}^{(1)}+\left(\Delta^{2}+2 \alpha \Delta_{3}\right) a_{-}^{(1)} \bar{a}_{+}^{(1)} \\
& +\Delta^{2} a_{3} \bar{a}_{3} \\
& +\left(\Delta^{2}-\alpha \Delta_{3}-\sqrt{3} \Delta_{8}\right) a_{+}^{(2)} \bar{a}_{-}^{(2)}+\left(\Delta^{2}+\alpha \Delta_{3}+\sqrt{3} \Delta_{8}\right) a_{-}^{(2)} \bar{a}_{+}^{(2)} \\
& +\left(\Delta^{2}+\alpha \Delta_{3}-\sqrt{3} \Delta_{8}\right) a_{+}^{(3)} \bar{a}_{-}^{(3)}+\left(\Delta^{2}-\alpha \Delta_{3}+\sqrt{3} \Delta_{8}\right) a_{-}^{(3)} \bar{a}_{+}^{(3)} \\
& +\Delta^{2} a_{8} \bar{a}_{8}
\end{aligned}
$$

where $\Delta^{2} \equiv\left(\Delta_{3}\right)^{2}+\left(\Delta_{8}\right)^{2}$ and

$$
a_{ \pm}^{(1)} \equiv \frac{1}{\sqrt{2}}\left(a_{1} \pm i a_{2}\right), a_{ \pm}^{(2)} \equiv \frac{1}{\sqrt{2}}\left(a_{4} \pm i a_{5}\right), \quad a_{ \pm}^{(3)} \equiv \frac{1}{\sqrt{2}}\left(a_{6} \pm i a_{7}\right) .
$$

The $N=2$ case is related to $\Delta_{8}=0$ in the above mass terms. We then have

$$
V_{2}=\left(\Delta_{3}^{2}-2 \alpha \Delta_{3}\right) a_{+}^{(1)} \bar{a}_{-}^{(1)}+\left(\Delta_{3}^{2}-\alpha \Delta_{3}\right) a_{+}^{(2)} \bar{a}_{-}^{(2)}+\left(\Delta_{3}^{2}-\alpha \Delta_{3}\right) a_{-}^{(3)} \bar{a}_{+}^{(3)} .
$$

The other components are massless or massive. Let us first investigate the situation $0<\Delta_{3}<\alpha$, where the three fields are tachyonic. After calculating the interaction terms, we can get the full potential,

$$
\begin{aligned}
V= & \left(\Delta_{3}^{2}-2 \alpha \Delta_{3}\right) t_{1}^{2}+\left(\Delta_{3}^{2}-\alpha \Delta_{3}\right) t_{2}^{2}+\left(\Delta_{3}^{2}-\alpha \Delta_{3}\right) t_{3}^{2} \\
& +t_{1}^{4}+t_{2}^{4}+t_{3}^{4}+2 t_{1}^{2} t_{2}^{2}+2 t_{2}^{2} t_{3}^{2}+2 t_{1}^{2} t_{3}^{2},
\end{aligned}
$$

where $a_{+}^{(1)}=t_{1} e^{i \theta_{1}}, a_{+}^{(2)}=t_{2} e^{i \theta_{3}}$ and $a_{+}^{(3)}=t_{3} e^{i \theta_{3}}$. The point $t_{1}=t_{2}=t_{3}=0$ corresponds to an unstable extremum. On the other hand, a stable extremum is found to be

$$
t_{1}^{2}=-\frac{1}{2} m_{1}^{2}, \quad t_{2}=t_{3}=0
$$

Although $t_{2}$ and $t_{3}$ are both zero, this point is stable. This situation is similar to the one encountered in equation (45). The terms $\left\langle t_{1}^{2}\right\rangle t_{2}^{2}$ and $\left\langle t_{1}^{2}\right\rangle t_{3}^{2}$ can respectively give the mass of $t_{2}$ and $t_{3}$. The stability of this point is found from $d^{2} V / d t_{1}^{2}=-2 m_{1}^{2}>0, d^{2} V / d t_{2}^{2}=d^{2} V / d t_{3}^{2}=2 \alpha \Delta_{3}>0$. The configuration which is realized in this case is similar to a fuzzy ellipsoidal sphere (28). When $\Delta_{3}$ takes the interval $\alpha \leq \Delta_{3}<2 \alpha$, only $a_{+}^{(1)}$ can be tachyonic. The tachyon potential is given by

$$
V=\left(\Delta_{3}^{2}-2 \Delta_{3}\right)\left(a_{+}^{(1)} \bar{a}_{-}^{(1)}\right)+\left(a_{+}^{(1)} \bar{a}_{-}^{(1)}\right)^{2} .
$$

When $\Delta_{3}$ is $\alpha$, a fuzzy sphere, which is constructed from the spin- $1 / 2$ representation of $S U(2)$, is obtained after tachyon condensation. This is the minimum of this potential.

The next analysis is devoted to three D0-branes. In this case, we can expect the formation both of the fuzzy $S^{2}$ and the fuzzy $C P^{2}$. If we consider the following background,

$$
\hat{x}_{3}=\alpha L_{3}^{(j=1)}, \quad \hat{x}_{i}=0(i \neq 3),
$$


we would obtain the spin-1 representation of $S U(2)$. Since this calculation is almost analogous to other cases, we omit the detail of it. The value of the classical potential for this solution is $V=-\alpha^{4}$.

We are next concerned with the following background:

$$
\hat{x}_{3}=\frac{\alpha}{2} \lambda_{3}, \quad \hat{x}_{8}=\frac{\alpha}{2} \lambda_{8}, \quad \hat{x}_{i}=0(i \neq 3,8) .
$$

The distances between these D0-branes are

$$
\begin{aligned}
& \Delta_{(12)}^{3}=\alpha, \quad \Delta_{(23)}^{3}=-\frac{1}{2} \alpha, \quad \Delta_{(13)}^{3}=\frac{1}{2} \alpha, \\
& \Delta_{(12)}^{8}=0, \quad \Delta_{(23)}^{8}=\frac{\sqrt{3}}{2} \alpha, \quad \Delta_{(13)}^{8}=\frac{\sqrt{3}}{2} \alpha .
\end{aligned}
$$

We parameterize the fluctuations around this solution as

$$
A_{\mu}=\left(\begin{array}{ccc}
0 & a_{\mu} & b_{\mu} \\
\bar{a}_{\mu} & 0 & c_{\mu} \\
\bar{b}_{\mu} & \bar{c}_{\mu} & 0
\end{array}\right) .
$$

The mass terms can be easily calculated by substituting (81) into (73) to give

$$
V_{2} / \alpha^{2}=-a_{+}^{(1)} \bar{a}_{-}^{(1)}-b_{+}^{(2)} \bar{b}_{-}^{(2)}-c_{+}^{(3)} \bar{c}_{-}^{(3)},
$$

where we have dropped non-tachyonic fields. The interaction terms for the tachyonic fields are calculated as follows:

$$
\begin{aligned}
V_{3}= & -\sqrt{2} \alpha\left(a_{+}^{(1)} \bar{b}_{+}^{(2)} c_{+}^{(3)}+\bar{a}_{+}^{(1)} b_{+}^{(2)} \bar{c}_{+}^{(3)}\right) \\
V_{4}= & \left(a_{+}^{(1)} \bar{a}_{-}^{(1)}\right)^{2}+\left(b_{+}^{(2)} \bar{b}_{-}^{(2)}\right)^{2}+\left(c_{+}^{(3)} \bar{c}_{-}^{(3)}\right)^{2} \\
& +\left(a_{+}^{(1)} \bar{a}_{-}^{(1)}\right)\left(b_{+}^{(2)} \bar{b}_{-}^{(2)}\right)+\left(b_{+}^{(2)} \bar{b}_{-}^{(2)}\right)\left(c_{+}^{(3)} \bar{c}_{-}^{(3)}\right)+\left(c_{+}^{(3)} \bar{c}_{-}^{(3)}\right)\left(a_{+}^{(1)} \bar{a}_{-}^{(1)}\right) .
\end{aligned}
$$

Note that $V_{3}$ is not zero, while it is zero in the $S U(2)$ background. Defining

$$
a_{+}^{(1)} \equiv t_{1} e^{i \theta_{1}}, \quad b_{+}^{(2)} \equiv t_{2} e^{i \theta_{2}}, \quad c_{+}^{(3)} \equiv t_{3} e^{i \theta_{3}},
$$

the extrema satisfy

$$
\begin{aligned}
& -2 t_{1}+4 t_{1}^{3}+2 t_{1} t_{2}^{2}+2 t_{1} t_{3}^{2}-2 \sqrt{2} t_{2} t_{3} \cos \left(\theta_{1}-\theta_{2}+\theta_{3}\right)=0, \\
& -2 t_{2}+4 t_{2}^{3}+2 t_{2} t_{1}^{2}+2 t_{2} t_{3}^{2}-2 \sqrt{2} t_{1} t_{3} \cos \left(\theta_{1}-\theta_{2}+\theta_{3}\right)=0, \\
& -2 t_{3}+4 t_{3}^{3}+2 t_{3} t_{1}^{2}+2 t_{3} t_{2}^{2}-2 \sqrt{2} t_{1} t_{2} \cos \left(\theta_{1}-\theta_{2}+\theta_{3}\right)=0 .
\end{aligned}
$$

$t_{1}=t_{2}=t_{3}=0$ is a trivial solution, representing an unstable extremum. The stable solution we are searching for is found to be $t_{1}=t_{2}=t_{3}=1 / \sqrt{2}$ and $\theta_{1}=\theta_{2}=\theta_{3}=0$. This solution provides the $(1,0)$ representation of $S U(3)$, that is to say, $X_{\mu}$ are represented as $\alpha \lambda_{\mu} / 2$. The value of the classical potential for this solution is $V=-\alpha^{4}$. For the $N=3$ case, we could obtain two noncommutative solutions. Although we can get other noncommutative solutions by changing the positions of the D0-branes, such noncommutative solutions do not minimize the 
classical potential. The classical energy of these two branes which we have obtained coincides accidentally. In general, the fuzzy two-sphere solution has lower classical energy than the fuzzy $C P^{2}$ solution. If we consider consider quantum effects, the transition between these solutions is expected to be seen.

It was shown in section 3 that a complex tachyon appears when a D0-brane approaches the surface of a spherical D2-brane, and that condensation of the tachyon produces a spherical D2brane with a larger radius. If we embed a system composed of a D0-brane and a D2-brane into eight-dimensional space under the $S U(3)$ invariant R-R four form background, we can observe a more interesting phenomenon. The condensation of tachyons appearing in this system produces a D4-brane which is constructed from the $S U(3)$ algebra. To observe such a phenomenon, let us start with the following classical solution:

$$
\begin{aligned}
& \hat{x}_{i}=\frac{\alpha}{2} \lambda_{i}=\frac{\alpha}{2}\left(\begin{array}{cc}
\sigma_{i} & 0 \\
0 & 0
\end{array}\right)(i=1,2,3), \\
& \hat{x}_{8}=d \frac{\alpha}{2} \lambda_{8}=\frac{\alpha}{2 \sqrt{3}}\left(\begin{array}{cc}
d \mathbf{1}_{2} & 0 \\
0 & -2 d
\end{array}\right) .
\end{aligned}
$$

The other coordinates are zero. The upper left part of the matrices represents a fuzzy two-sphere whose position is $\left(x_{3}, x_{8}\right)=(0, \alpha d / 2 \sqrt{3})$, and the lower right part a D0-brane whose position is $(0,-\alpha d / \sqrt{3})$. If $d$ is infinity, these two branes are far away in the eighth direction, and there do not appear any tachyonic modes from strings connecting these two branes. When $d$ takes a value, we can show that tachyonic fields appear. The mass term is evaluated as

$$
\begin{aligned}
& V_{2} / \alpha^{2}=\frac{3}{4}\left(d^{2}+1\right) v_{\mu}^{\dagger} v_{\mu} \\
& +\frac{3}{2} i d\left(v_{5}^{\dagger} v_{4}-v_{4}^{\dagger} v_{5}+v_{7}^{\dagger} v_{6}-v_{6}^{\dagger} v_{7}\right)+\frac{1}{2} i d\left(v_{5}^{\dagger} \sigma_{3} v_{4}-v_{4}^{\dagger} \sigma_{3} v_{5}+v_{7}^{\dagger} \sigma_{3} v_{6}-v_{6}^{\dagger} \sigma_{3} v_{7}\right) \\
& -\frac{1}{2} i\left(v_{4}^{\dagger} \sigma_{1} v_{7}-v_{7}^{\dagger} \sigma_{1} v_{4}-v_{5}^{\dagger} \sigma_{1} v_{6}+v_{6}^{\dagger} \sigma_{1} v_{5}+v_{4}^{\dagger} \sigma_{2} v_{6}-v_{6}^{\dagger} \sigma_{2} v_{4}+v_{5}^{\dagger} \sigma_{2} v_{7}-v_{7}^{\dagger} \sigma_{2} v_{5}\right),
\end{aligned}
$$

where $v_{\mu}$ and $v_{\mu}^{\dagger}$ are off-diagonal fluctuations. It can be shown that the tachyonic modes do not appear from $v_{i}(i=1,2,3,8)$, the second component of $v_{4}, v_{5}$ and the first component of $v_{6}, v_{7}$. Therefore, we make them zero. In the remainder of this calculation, $v_{4}$ and $v_{5}$ denote their first components, and $v_{6}$ and $v_{7}$ their second components. The mass terms for $v_{4}, v_{5}, v_{6}$ and $v_{7}$ are diagonalized as follows,

$$
\begin{aligned}
V_{2} / \alpha^{2} & =\left(\frac{3}{4} d^{2}-2 d-\frac{1}{4}\right) \frac{1}{2}\left(v_{+}^{(2)}+v_{+}^{(3)}\right)\left(\bar{v}_{-}^{(2)}+\bar{v}_{-}^{(3)}\right) \\
& +\left(\frac{3}{4} d^{2}-2 d+\frac{7}{4}\right) \frac{1}{2}\left(v_{+}^{(2)}-v_{+}^{(3)}\right)\left(\bar{v}_{-}^{(2)}-\bar{v}_{-}^{(3)}\right) \\
& +\left(\frac{3}{4} d^{2}+2 d+\frac{3}{4}\right) \frac{1}{2} v_{-}^{(2)} \bar{v}_{+}^{(2)}+\left(\frac{3}{4} d^{2}+2 d+\frac{3}{4}\right) \frac{1}{2} v_{-}^{(3)} \bar{v}_{+}^{(3)},
\end{aligned}
$$

where

$$
v_{ \pm}^{(2)}=\frac{1}{\sqrt{2}}\left(v_{4} \pm v_{5}\right), \quad v_{ \pm}^{(3)}=\frac{1}{\sqrt{2}}\left(v_{6} \pm v_{7}\right)
$$


Only the first term can be a tachyonic mass term when $d$ takes the following interval,

$$
\frac{4}{3}-\frac{\sqrt{19}}{3}<d<\frac{4}{3}+\frac{\sqrt{19}}{3} .
$$

This instability is different from one we confronted in the section 3 or Appendix B which happens when a D0-brane approaches the surface of a fuzzy space. This instability happens when the distance between two branes in the eighth direction takes a value. It is interesting to consider $d=1$. Only the first term in (89) is relevant to this case. The full tachyon potential in this case is

$$
V=-3 t^{2}+3 t^{4}
$$

where $v_{+}^{(2)}=v_{+}^{(3)}=t e^{i \theta}$. The end point of tachyon condensation is given by $t=1 / \sqrt{2}$, where the fuzzy $C P^{2}$ given by the $(1,0)$ representation of $S U(3)$ is realized.

\section{$6 \quad$ Summary and discussions}

In this work, we studied tachyon condensation in the process of the Myers effect by using the low energy effective Yang-Mills action. D0-branes under the R-R field strength background are unstable, and we can expect that this system can become stable by the condensation of tachyon. We have considered two kinds of R-R field strength, one being given by the structure constant of $S U(2)$, and the other, by that of $S U(3)$. The characteristic configurations of these cases are the fuzzy sphere and the fuzzy $C P^{2}$ respectively.

In general, a classical solution given by a reducible representation is unstable, and it is the irreducible representation that minimizes the classical potential. We explicitly confirmed that the instability of the reducible representations is manifested by the existence of tachyonic fields, and that the irreducible representation is realized after the condensation of the tachyons. A big difference between $S U(2)$ and $S U(3)$ is that the size of their matrices is restricted in the latter case as in (65), while it is not in the former case. For the $S U(2)$ invariant background case, the only stable configuration is a fuzzy sphere whose coordinate is given by the irreducible representation of $S U(2)$. The fact that the reducible representation of $S U(2)$ rolls down to the irreducible representation of $S U(2)$ does not depend on the dimension of the representation. On the other hand, this is not the case when we consider the reducible representation of $S U(3)$. Although the formation of the irreducible representation of $S U(3)$ from the reducible representation of $S U(3)$ is not always seen, tachyonic instability always appears when the reducible representation is considered. ${ }^{5}$ Since runaway behavior never happens from the shape of the potential, a noncommutative solution can expected to appear. ${ }^{6}$

An interesting property of matrix models or noncommutative gauge theories is background independence [46]. The matrix model variables are $N \times N$ matrices $X_{\mu}$. Different D-branes appear as different classical solutions of matrix models. The fields on the branes can be described

\footnotetext{
${ }^{5}$ It is shown in the Appendix $\mathbb{B}$ that a reducible representation which is composed of a D0-brane and a fuzzy $C P^{2}$ is unstable due to the existence of a complex tachyonic field. This proof can be extended to general reducible representations of $S U(3)$. We should not overlook that this discussion is independent of the size of the representation.

${ }^{6}$ Such an idea has already been applied in [45].
} 
by expanding $X_{\mu}$ around the classical solutions as given in equation (14). We have treated in this study a simple matrix model action with a Chern-Simons term. Different curved D-branes arose as classical solutions, and the condensation of tachyons led to the transition between different D-branes.

As shown in the section [5 a fuzzy $C P^{2}$ is formed from a D0-brane and a fuzzy $S^{2}$ through the condensation of tachyon. A similar phenomenon such as the formation of the fuzzy $C P^{2}$ from the fuzzy $C P^{2}$ and the fuzzy $S^{2}$ can be seen. The important thing leading to these phenomena is that the matrix representation of $S U(2)$ is included in that of $S U(3)$. In general, we can say that D-branes whose coordinates are given by higher dimensional Lie-algebra shows interesting dynamics. In this sense, it is also an interesting problem to study the higher dimensional fuzzy sphere [47, 48, in the context of the tachyon condensation.

\section{Acknowledgments}

I acknowledge helpful discussions with T. Masuda. I also thank colleagues at RIKEN for some discussions.

\section{A $(m, n)$ representation of $S U(3)$}

In this Appendix, we construct some formulae which are relevant to the $(m, n)$ representation of $S U(3) . T_{\mu}^{(m, n)}$ denotes the generator of $(m, n)$ representation, being constructed as

$$
\begin{aligned}
T_{\mu}^{(m, n)=} & \left(t_{\mu} \otimes 1 \otimes \cdots \otimes 1+1 \otimes t_{\mu} \otimes \cdots \otimes 1+\cdots+1 \otimes \cdots \otimes t_{\mu} \otimes \cdots \otimes 1\right. \\
& \left.+1 \otimes \cdots \otimes 1 \otimes s_{\mu} \otimes \cdots \otimes 1+\cdots+1 \otimes \cdots \otimes 1 \otimes s_{\mu}\right),
\end{aligned}
$$

where $t_{\mu}$ and $s_{\mu}=-t_{\mu}^{*}$ are the generators of fundamental and anti-fundamental representation respectively. $t_{\mu}$ is written as $\lambda_{\mu} / 2$ using the Gell-Mann matrix. $T_{\mu}^{(m, n)}$ is assumed to act on the following state,

$$
\left|a_{1}, a_{2}, \ldots, a_{m}, \bar{a}_{m+1}, \ldots, \bar{a}_{m+n}\right\rangle .
$$

where this state has the same symmetry as the Young diagram corresponding to the $(m, n)$ representation of $S U(3)$. The dimension of this sate is given by (65).

We provide some useful formulae,

$$
\begin{aligned}
& \sum_{\mu=1}^{8}\left(t_{\mu} \otimes t_{\mu}\right)=\frac{1}{3}(1 \otimes 1), \\
& \sum_{\mu=1}^{8}\left(t_{\mu} t_{\nu} \otimes t_{\mu}\right)=\frac{1}{3}\left(t_{\nu} \otimes 1\right), \quad \sum_{\mu=1}^{8}\left(t_{\nu} t_{\mu} \otimes t_{\mu}\right)=\frac{1}{3}\left(t_{\nu} \otimes 1\right) .
\end{aligned}
$$

These are easily proved by using

$$
\sum_{\mu=1}^{8}\left(t_{\mu}\right)_{i j}\left(t_{\mu}\right)_{k l}=\frac{1}{2} \delta_{i l} \delta_{j k}-\frac{1}{6} \delta_{i j} \delta_{k l} .
$$


We also provide

$$
\sum_{\mu=1}^{8}\left(t_{\mu} \otimes s_{\mu}\right)=\frac{1}{6}(1 \otimes 1) .
$$

The quadratic Casimir is easily calculated by using the above formulae,

$$
C_{2}(m, n)=T_{\mu}^{(m, n)} T_{\mu}^{(m, n)}=\frac{1}{3}\left(m^{2}+3 m+n^{2}+3 n+m n\right) 1_{N} .
$$

\section{B Instability of curved branes}

In this Appendix, we observe the instability of reducible representations. We consider a system of a fuzzy brane and a D0-brane as a simple example of reducible representations. Tachyonic modes appearing from strings connecting a fuzzy brane and a D0-brane manifest the instability of this system.

We first show the instability of D0-D2 system. The detail of this calculation is found in [17]. The configuration of a D0-brane and a spherical D2-brane is represented by

$$
L_{\mu}=\left(\begin{array}{cc}
c_{\mu} / \alpha & 0 \\
0 & L_{\mu}^{(j)}
\end{array}\right),
$$

where $c_{\mu}$ is a number and $L_{\mu}^{(j)}$ is the spin $j \equiv(N-2) / 2$ representation of $S U(2)$. A complex tachyon appears from off diagonal parts of the fluctuations,

$$
A_{\mu}=\left(\begin{array}{cc}
0 & v_{\mu} \\
v_{\mu}^{\dagger} & 0
\end{array}\right),
$$

where $v_{\mu}$ are $1 \times(N-1)$ matrices. Mass terms for $v_{\mu}$ are calculated as

$$
\begin{aligned}
V_{2} / \alpha^{2}= & \left(c^{2}-2 c-2 c m+j(j+1)\right) v_{+} v_{-}^{\dagger}+\left(c^{2}+2 c-2 c m+j(j+1)\right) v_{-} v_{+}^{\dagger} \\
& +\left(c^{2}-2 c m+j(j+1)\right) v_{3} v_{3}^{\dagger},
\end{aligned}
$$

where $m$ runs over $-j$ to $j$. We have used the rotation symmetry to fix $c_{\mu}$ as $c \delta_{\mu 3}$, which does not lose generality. If we suppose $c \geq 0$, only an $m=j$ component of $v_{+}$( or $v_{-}^{\dagger}$ ) becomes tachyonic when the position of the D0-brane takes the following interval,

$$
j+1-\sqrt{j+1}<\frac{c}{\alpha}<j+1+\sqrt{j+1} .
$$

This shows that a complex tachyon appears when a D0-brane is close to the surface of a spherical D2-brane. This manifests the instability of the system, and after the tachyon condensation this system is expected to become the irreducible representation which is given by the spin $(N-1) / 2$ representation of $S U(2)$.

We next show the instability of D0-D4 system where a D4-brane forms fuzzy $C P^{2}$. This calculation is analogous to the previous D0-D2 system. A classical solution representing such a system is given by

$$
J_{\mu}=\left(\begin{array}{cc}
c_{\mu} / \alpha & 0 \\
0 & T_{\mu}^{(m, n)}
\end{array}\right),
$$


where $c_{\mu}$ are the positions of a D0-brane and $T_{\mu}^{(m, n)}(m \geq n)$ are the $(m, n)$ representation of $S U(3)$. The size of the matrices $J_{\mu}$ is $N+1$ where $N$ is given by (65). The mass term is calculated as

$$
V_{2} / \alpha^{2}=v_{\nu}^{\dagger}\left(T_{\mu}^{(m, n)}-c_{\mu} 1_{N}\right)\left(T_{\mu}^{(m, n)}-c_{\mu} 1_{N}\right) v_{\nu}+2 i f_{\mu \nu \lambda} v_{\mu}^{\dagger} v_{\nu} c_{\lambda} .
$$

Let us consider the case $c_{\mu}=\left(0,0, c_{1}, 0,0,0,0, c_{2}\right)$. The above mass terms become

$$
\begin{aligned}
V_{2} / \alpha^{2}= & \left(c_{1}^{2}+c_{2}^{2}-2\left(c_{1} t_{3}+c_{2} t_{8}\right)+C_{2}(m, n)\right) v_{\mu}^{\dagger} v_{\mu} \\
& +2 i c_{1}\left(v_{1}^{\dagger} v_{2}-v_{2}^{\dagger} v_{1}\right)+i c_{1}\left(v_{4}^{\dagger} v_{5}-v_{5}^{\dagger} v_{4}-v_{6}^{\dagger} v_{7}+v_{7}^{\dagger} v_{6}\right) \\
& +\sqrt{3} i c_{2}\left(v_{4}^{\dagger} v_{5}-v_{5}^{\dagger} v_{4}+v_{6}^{\dagger} v_{7}-v_{7}^{\dagger} v_{6}\right) .
\end{aligned}
$$

In this expression, $t_{3}$ and $t_{8}$ denote eigenvalues of $T_{3}^{(m, n)}$ and $T_{8}^{(m, n)}$ respectively. These mass terms are diagonalized as

$$
\begin{aligned}
V_{2} / \alpha^{2}= & m_{1}^{2}\left(v_{3}^{\dagger} v_{3}+v_{8}^{\dagger} v_{8}\right)+m_{2+}^{2} v_{-}^{(1) \dagger} v_{+}^{(1)}+m_{2-}^{2} v_{+}^{(1) \dagger} v_{-}^{(1)} \\
& +m_{3+}^{2} v_{-}^{(2) \dagger} v_{+}^{(2)}+m_{3-}^{2} v_{+}^{(2) \dagger} v_{-}^{(2)}+m_{4+}^{2} v_{-}^{(3) \dagger} v_{+}^{(3)}+m_{4-}^{2} v_{+}^{(3) \dagger} v_{-}^{(3)},
\end{aligned}
$$

where the mass is given by

$$
\begin{aligned}
& m_{1}^{2}=c_{1}^{2}+c_{2}^{2}-2\left(c_{1} t_{3}+c_{2} t_{8}\right)+C_{2}, \\
& m_{2 \pm}^{2}=c_{1}^{2}+c_{2}^{2}-2\left(c_{1} t_{3}+c_{2} t_{8}\right)+C_{2} \pm 2 c_{1}, \\
& m_{3 \pm}^{2}=c_{1}^{2}+c_{2}^{2}-2\left(c_{1} t_{3}+c_{2} t_{8}\right)+C_{2} \pm\left(c_{1}+\sqrt{3} c_{2}\right) \\
& m_{4 \pm}^{2}=c_{1}^{2}+c_{2}^{2}-2\left(c_{1} t_{3}+c_{2} t_{8}\right)+C_{2} \pm\left(-c_{1}+\sqrt{3} c_{2}\right) .
\end{aligned}
$$

We find after careful consideration that $m_{1}^{2}, m_{2 \pm}^{2}, m_{3-}^{2}$ and $m_{4-}^{2}$ are always positive. We can show that $m_{3+}^{2}$ and $m_{4+}^{2}$ can be negative only if $\left(t_{8}, t_{3}\right)=(-(m+n / 2) / \sqrt{3},-n / 2)$ and $(-(m+$ $n / 2) / \sqrt{3}, n / 2)$ respectively. The value of $t_{8}$ corresponds to the minimal value of $T_{8}^{(m, n)}$ (see (67)). As calculated in (68), a fuzzy two-sphere is located in this point, which is given by the spin $n / 2$ representation of $S U(2)$. Therefore $t_{3}=+n / 2,-n / 2$ are the maximal and minimal values of $T_{3}^{(m, n)}$. The inequality we need to solve is

$$
\begin{aligned}
m_{3+, 4+}^{2} & =c_{1}^{2}-\left(2 t_{3} \mp 1\right) c_{1}+c_{2}^{2}-\left(2 t_{8}-\sqrt{3}\right) c_{2}+C_{2} \\
& =\left(c_{1} \pm \frac{n+1}{2}\right)^{2}+\left(c_{2}+\left(\frac{m}{3}+\frac{n}{6}+\frac{1}{2}\right) \sqrt{3}\right)-1<0
\end{aligned}
$$

where we have substituted $t_{8}=-(m+n / 2) / \sqrt{3}$ and $t_{3}=\mp n / 2$. The expressions of $m_{3+}^{2}$ and $m_{4+}^{2}$ are exchanged by changing the sign of $c_{1}$. We first regard this inequality as the second order one of $c_{1}$, it being solved as

$$
\mp \frac{n+1}{2}-\sqrt{D}<\frac{c_{1}}{\alpha}<\mp \frac{n+1}{2}+\sqrt{D},
$$

where $D$ satisfies

$$
0<D \leq \frac{n}{2}+\frac{7}{4}
$$


The condition $D>0$ gives

$$
-\frac{m+\frac{n}{2}}{\sqrt{3}}-\frac{\sqrt{3}}{2}-\sqrt{\frac{n}{2}+\frac{7}{4}}<\frac{c_{2}}{\alpha}<-\frac{m+\frac{n}{2}}{\sqrt{3}}-\frac{\sqrt{3}}{2}+\sqrt{\frac{n}{2}+\frac{7}{4}} .
$$

We have shown that a complex tachyon appears when a D0-brane approaches the surface of a fuzzy $C P^{2}$. We would like to emphasize that the equation (B.11) reflects the fact that the fuzzy $C P^{2}$ has the extension along a fuzzy two-sphere (see [68) ).

\section{References}

[1] J. Polchinski, TASI Lectures on D-Branes, hep-th/9611050.

[2] W. Taylor, Lectures on D-branes, Gauge Theory and M(atrices), hep-th/9801182

[3] E. Witten, Bound States Of Strings And p-Branes, Nucl.Phys.B460 (1996) 335, hep-th/9510135.

[4] N. Seiberg and E. Witten, String theory and Noncommutative Geometry, JHEP9909(1999)032, hep-th/9908142.

[5] T. Banks, W. Fischler, S. Shenker and L. Susskind, M theory as a matrix model: a conjecture, Phys.Rev.D55 (1997) 5112, hep-th/9610043.

[6] N. Ishibashi, H. Kawai, Y. Kitazawa and A. Tsuchiya, A Large $N$ reduced model as superstring, Nucl.Phys.B498 (1997) 467, hep-th/9612115.

[7] A. Connes, M. Douglas and A. Schwarz, Noncommutative Geometry and Matrix Theory : Compactification on Tori, JHEP 9802 (1998) 003, hep-th/9711162.

[8] H. Aoki, N. Ishibashi, S. Iso, H. Kawai, Y. Kitazawa and T. Tada, Noncommutative YangMills in IIB Matrix Model, Nucl.Phys. B565 (2000) 176, hep-th/9908141

[9] J. Ambjorn, Y.M. Makeenko, J. Nishimura and R.J. Szabo, Finite N Matrix Models of Noncommutative Gauge Theory JHEP 9911 (1999) 029, hep-th/9911041.

[10] R. Myers, Dielectric-Branes, JHEP9912 (1999) 022, hep-th/9910053.

[11] J. Hoppe, Quantum Theory of A Massless Relativistic Surface and A Two-Dimensional Bound State Problem, MIT Ph.D.Thesis,1982.

[12] J. Madore, The fuzzy sphere, Class.Quant.Grav.9 (1992) 69.

[13] D. Kabat and W. Taylor, Spherical membranes in Matrix theory, Adv.Theor.Math.Phys. 2 (1998) 181, hep-th/9711078.

[14] U. Carow-Watamura and S. Watamura, Noncommutative Geometry and Gauge Theory on Fuzzy Sphere, Comm.Math.Phys. 212 (2000) 395, hep-th/9801195. 
[15] A. Alekseev, A. Recknagel and V. Schomerus, Non-commutative World-volume Geometries: Branes on SU(2) and Fuzzy Spheres, JHEP 9909 (1999) 023, hep-th/9908040.

[16] S. Iso, Y. Kimura, K. Tanaka and K. Wakatsuki, Noncommutative Gauge Theory on Fuzzy Sphere from Matrix Model, Nucl.Phys. B604 (2001) 121, hep-th/0101102.

[17] K. Hashimoto and K. Krasnov, D-brane Solutions in Non-Commutative Gauge Theory on Fuzzy Sphere, Phys.Rev.D64 (2001) 046007, hep-th/0101145.

[18] D. Jatkar, G. Mandal, S. Wadia and K. Yogendran, Matrix dynamics of fuzzy spheres, JHEP0201 (2002) 039, hep-th/0110172.

[19] Y. Hikida, M. Nozaki and Y. Sugawara, Formation of Spherical D2-brane from Multiple D0-branes, Nucl.Phys.B617 (2001) 117, hep-th/0101211.

[20] Y. Gao and Z. Yang, Interactions between Dielectric Branes, JHEP0105 (2001) 018, hep-th/0102151.

[21] Y. Hyakutake, Torus-like Dielectric D2-brane, JHEP 0105 (2001) 013, hep-th/0103146.

[22] S. Bal and H. Takata, Interaction between two Fuzzy Spheres, Int.J.Mod.Phys.A17 (2002) 2445, hep-th/0108002.

[23] K. Hashimoto, Dynamical Decay of Brane-Antibrane and Dielectric Brane, JHEP 0207 (2002) 035, hep-th/0204203

[24] T. Imai, Y. Kitazawa, Y. Takayama and D. Tomino, Quantum Corrections on Fuzzy Sphere, hep-th/0303120.

[25] J. Pawelczyk and H. Steinacker, Algebraic brane dynamics on SU(2): excitation spectra, hep-th/0305226.

[26] A. Sen, Universality of the Tachyon Potential, JHEP 9912 (1999) 027, hep-th/9911116. Tachyon Condensation on the Brane Antibrane System, JHEP 9808 (1998) 012, hep-th/9805170 Non-BPS States and Branes in String Theory, hep-th/9904207.

[27] K. Bardakci, Dual Models and Spontaneous Symmetry Breaking, Nucl. Phys. B68 (1974) 331; Spontaneous Symmetry Breakdown in the Standard Dual String Model, Nucl. Phys. B133 (1978) 297.

K. Bardakci and M.B. Halpern, Explicit Spontaneous Breakdown in a Dual Model, Phys. Rev. D10 (1974) 4230; Explicit Spontaneous Breakdown in a Dual Model II: N Point Functions, Nucl. Phys. B96 (1975) 285.

[28] A. Hashimoto and W. Taylor IV, Fluctuation Spectra of Tilted and Intersecting D-branes from the Born-Infeld Action, Nucl.Phys. B503 (1997) 193, hep-th/9703217.

[29] E. Gava, K.S. Narain and M.H. Sarmadi, On the Bound States of $p$ - and $(p+2)$-Branes, Nucl.Phys. B504 (1997) 214, hep-th/9704006. 
[30] H. Awata, S. Hirano and Y. Hyakutake, Tachyon Condensation and Graviton Production in Matrix Theory, hep-th/9902158.

[31] M. Massar and J. Troost, The longitudinal fivebrane and tachyon condensation in matrix theory Nucl.Phys. B569 (2000) 417, hep-th/9907128

[32] M. Aganagic, R. Gopakumar, S. Minwalla and A. Strominger, Unstable Solitons in Noncommutative Gauge Theory, JHEP 0104 (2001) 001, hep-th/0009142.

[33] P. Kraus, A. Rajaraman and S. Shenker, Tachyon Condensation in Noncommutative Gauge Theory, Nucl.Phys. B598 (2001) 169, hep-th/0010016

[34] G. Mandal and S. R. Wadia, Matrix Model, Noncommutative Gauge Theory and the Tachyon Potential, Nucl.Phys. B599 (2001) 137, hep-th/0011094.

[35] K. Hashimoto and S. Nagaoka Recombination of Intersecting D-branes by Local Tachyon Condensation, hep-th/0303204.

[36] V.P. Nair and S. Randjbar-Daemi, On brane solutions in M(atrix) theory, Nucl.Phys. B533 (1998) 333, hep-th/9802187.

[37] S. P. Trivedi and S. Vaidya, Fuzzy Cosets and their Gravity Duals, JHEP0009 (2000) 041, hep-th/0007011.

[38] G. Alexanian, A. P. Balachandran, G. Immirzi and B. Ydri, Fuzzy CP2, J.Geom.Phys.42 (2002) 28, hep-th/0103023.

[39] B. Ydri, Fuzzy Physics, hep-th/0110006.

[40] D. Karabali and V. P. Nair Quantum Hall Effect in Higher Dimensions, Nucl.Phys.B641 (2002) 533, hep-th/0203264.

[41] G.G. Alexanian, A.P. Balachandran and P.J. Silva, Obstruction to D-brane topology change, hep-th/0207052.

[42] Y. Kitazawa, Matirix Models in Homogeneous Spaces, Nucl.Phys.B642 (2002) 210, hep-th/0207115.

[43] J. Pawelczyk and H. Steinacker, A quantum algebraic description of D-branes on group manifolds, Nucl.Phys. B638 (2002) 433, hep-th/0203110.

[44] S. Aoyama and T. Masuda, Fuzzy Algebrae of the General Kaehler Coset Space $G / H \otimes U(1)^{k}$, Mod.Phys.Lett. A18 (2003) 553, hep-th/0209082.

[45] J. Boer, E. Gimon, K. Schalm and J. Wijnhout, Evidence for a gravitational Myers effect, hep-th/0212250.

[46] N. Seiberg, A Note on Background Independence in Noncommutative Gauge Theories, Matrix Model and Tachyon Condensation, JHEP 0009 (2000) 003, hep-th/0008013. 
[47] P.M. Ho and S. Ramgoolam, Higher Dimensional Geometries from Matrix Brane constructions, Nucl.Phys. B627 (2002) 266, hep-th/0111278.

[48] Y. Kimura, Noncommutative Gauge Theory on Fuzzy Four-Sphere and Matrix Model, Nucl.Phys. B637 (2002) 177, hep-th/0204256, On Higher Dimensional Fuzzy Spherical Branes, Nucl.Phys. B664 (2003) 512, hep-th/0301055 